\title{
Convivencia escolar y solución de conflictos mediadas por la tecnología de la información y comunicación ${ }^{1}$ \\ School coexistence and conflict resolution mediated by information and communication technology
}

\author{
DOI: http://dx.doi.org/10.17981/cultedusoc.9.1.2018.10
}

Fecha de recepción: 21/04/2018. Fecha de aceptación: 19/07/2018

Edgar Ramón Camacho-Amaya ${ }^{2}$ iD

Arelis María Fonseca-Brochero; Marlene Isabel Mercado; Yaneth María Escorcia-Polo; Yasnuris Rocío Gómez-López; Martha Elena Ariza-Álvarez; Juan Vicente Gómez-Colón; William Harry de La Hoz-Cera; Nubia Visbal de Marín ${ }^{3}$

\begin{abstract}
Para citar este artículo
Camacho-Amaya, E., Fonseca-Brochero, A., Mercado, M., Escorcia-Polo, Y., Gómez-López, Y., Ariza-Álvarez, M., Gómez-Colón, J., De La Hoz-Cera, W. y Visbal, N. (2018). Convivencia escolar y solución de conflictos mediadas por la tecnología de la información y comunicación. Cultura. Educación y Sociedad 9(1), 134-146. DOI: http://dx.doi.org/10.17981/cultedusoc.9.1.2018.10
\end{abstract}

\section{Resumen}

Colombia es un país que se está preparando para la paz, sin embargo, en las instituciones educativas se encuentran altos índices de conflictos, por lo cual es imperante emplear estrategias educativas que fomenten una sana convivencia. El articulo tiene como objetivo presentar estrategias pedagógicas para fortalecer la convivencia escolar y solución de conflictos, mediante el uso de tecnologías de la información y la comunicación. El estudio es de tipo cualitativo bajo el modelo de investigación acción. Se evidenciaron manifestaciones violentas y comportamientos erráticos por parte de los estudiantes en la interacción con su grupo de pares, donde se demuestran agresiones verbales, físicas, discriminación, lenguaje soez, ruptura de normas escolares e irrespeto a la autoridad, la cual es representada por docentes y cuerpo administrativo de la institución, donde a través de las TIC se crearon espacios de participación que permitieron generar una mejor convivencia escolar basada en la solución de conflictos.

Palabras clave: convivencia escolar, solución de conflictos, tecnología de la información y comunicación.

\begin{abstract}
Colombia is a country that is preparing for peace, however, in educational institutions are high rates of conflict, which is why it is imperative to use educational strategies that promote a healthy coexistence. The aim of the article is to present pedagogical strategies to strengthen school coexistence and conflict resolution, through the use of information and communication technologies. The study is of qualitative type under the action research model. There were violent demonstrations and erratic behavior by students in the interaction with their peer group, where verbal, physical aggressions, discrimination, foul language, breaking of school rules and disrespect to the authority are shown, which is represented by teachers. and the administrative body of the institution, where through TIC spaces of participation were created that allowed generating a better school coexistence based on the solution of conflicts.
\end{abstract}

Keywords: school coexistence, conflict resolution, information and communication technology.

\footnotetext{
${ }^{1}$ Este artículo ha sido derivado del Programa de Fortalecimiento de la Cultura Ciudadana y Democrática CT+I a través de la IEP apoyada en TIC en el Departamento de Magdalena: CICLON

${ }^{2}$ Docente de la Institución Educativa John F. Kennedy y líder del grupo de investigación Unidos Holmes Kennedistas. Correo de correspondencia: ercam64@hotmail.com

${ }^{3}$ Docentes de la Institución Educativa John F. Kennedy, Sede Cauca y miembros del grupo de investigación Unidos Holmes Kennedistas.
}

- The author; licensee Universidad de la Costa - CUC. 


\section{Introducción}

En la actualidad existen investigaciones en el ámbito de la psicología educacional, que abordan el tema de la convivencia desde diversos ángulos, en los cuales se pone de relieve la importancia de los contextos normativos institucionales, que tienden a favorecer el establecimiento de distintos conflictos relacionados con las estructuras de poder y los roles que se configuran al interior de las escuelas. Cabe decir que, durante los últimos años, los diversos estudios en ciencias sociales relacionados al sistema educacional en América Latina, han dado cuenta de una convivencia escolar compleja y en crisis, especialmente en aquellos liceos donde las condiciones ambientales, la escasez de recursos y el contexto en que éstos se insertan debilitar las dificultades de interacción que la educación pública debe ir superando para mantener su vigencia a través del tiempo.

La convivencia escolar y solución de conflictos mediadas por la tecnología de la información y comunicación, consiste en gran medida en compartir, y al compartir se aprende, en los tiempos, espacios, logros, dificultades, proyectos y sueños. El aprendizaje de valores y habilidades sociales, así como las buenas prácticas de convivencia, son la base del futuro ciudadano, y ese aprendizaje tiene lugar en gran medida en la experiencia escolar. Todos los sujetos que participan del sistema de interrelaciones que se establecen en una comunidad educativa, cumplen un rol y tienen responsabilidad en el tipo de convivencia que existe en la institución educativa, así como en el proceso de enseñanza-aprendizaje de dicha convivencia.

Algunas personas asocian el termino de convivencia escolar a un ambiente cá- lido, de relaciones humanas amables y no violentas; mientras otros asocian buena convivencia a un ambiente disciplinado entendiendo por tal ordenado, respetuoso, obediente al quehacer escolar. (Mena, y Huneeus, 2017).

Se plantea desde los antes en mención, que los esfuerzos por posicionar a la escuela como una de las instituciones socializadoras más relevantes en la formación social y personal de los individuos, han sido tarea de este y otros gobiernos. En la historia, los temas relevantes para forjar las políticas educativas que contribuyan al desarrollo cultural, han ido transformándose según los distintos lugares que han ocupado en los proyectos de gobierno y sociedad. De acuerdo a lo anterior, el sistema educativo actualmente, no puede asegurar cuál perfil de profesor logrará mejores resultados en términos de aprendizajes y qué estudiantes tendrán mayores oportunidades de inserción laboral, porque la dinámica que se instaura en el proceso mismo de la educación y que da lugar a desigualdades en los resultados es poco conocida, (Cassasus, 2003).

En otras palabras, se plantea una forma de dar cuenta de los procesos de construcción y choque de sentido que se manifiestan en la relación de ambos actores de la educación, es indagar estratégicamente como lugar de estudio en la sala de clases. Podríamos señalar que es en el escenario institucional llamado aula, el espacio concreto donde formalmente una cultura escolar particular pone de manifiesto o no una serie de normas complejas que enmarcan y definen el rol de alumnos y profesores dentro de una organización jerarquizada, teniendo en cuenta las condiciones socioeconómicas y socioculturales tanto de los estudiantes, como del educador. 
En efecto, se plantea como un reto para las ciencias sociales en la medida en que puedan ir generando un cuerpo de conocimientos que, desde la investigación/intervención, vayan develando de qué forma los actores se ven involucrados y también aquellos factores que generan logros y, por tanto, más calidad en la educación. Esta figura educativa no deja de ser central en la generación de una sana convivencia, ya que cumple un rol fundamental en facilitar las condiciones necesarias para que se les otorgue a los jóvenes un espacio donde puedan ir tomando conciencia de sí mismos y su desarrollo.

Cabe decir que, el conflicto escolar circula a ser el punto de crisis de la convivencia, donde dos fuerzas se contraponen; lo que cuestiona, detiene o rompe el esquema de convivencia anterior, que es el regular. Este se convierte en un tema emergente y de importancia psicológica al asociarlo al grupo curso y a los grupos que se forman al intrínseco del mismo ya que son espacios de identidad y referencia que se resisten al cambio.

Asimismo, en un grupo humano tan amplio y con actores tan diversos es probable que el conflicto surja con frecuencia, ya que se relaciona con las necesidades no satisfechas y la ley que las restringe o regula, por lo cual, su desafío y/o resolución, permiten que la institución supere los diversos obstáculos internos que presenta y que aparecen como incontrolables, pero que desde esta dirección pueden ser manejables e incluso constructivos. Es aquí donde surge la relevancia de abordar el conflicto dentro de la escuela, ya que en ella se establecen interrelaciones entre distintos actores, donde unos intentan transmitir el saber a otros, es decir, es la apropiación del conocimiento y el aprendizaje lo que está mediando estas relaciones.
La sociedad colombiana enfrenta retos en lo económico, social, político y cultural, para construir un país abierto a las nuevas realidades de la ciencia y el conocimiento, las cuales exigen el desarrollo $\mathrm{y}$ fomento de capacidades para que sus ciudadanos puedan afrontar los cambios de estos tiempos. Ante tal premisa se hace necesario presentar estrategias pedagógicas para fortalecer la convivencia escolar y solución de conflictos, mediante el uso de tecnologías de la información y la comunicación (TIC) en básica primaria, a fin de atender a las necesidades de la población y resuelva sus problemas, teniendo en cuenta las potencialidades naturales, culturales y particularidades de la región.

Así mismo, es urgente construir una convivencia plena, lo que sólo será posible si la mayoría de los colombianos accede a los procesos de generación de conocimiento y saber, participando en ellos a través de mecanismos que les permitan interesarse, comprenderlos, validarlos y reconocerlos operando en su vida cotidiana; es decir, formarse una opinión al respecto y participar como ciudadanos plenos en la toma de decisiones sobre su vida, sobre los problemas que los afecten directa o indirectamente y sobre todos los aspectos de la existencia en los que incidan la ciencia y la tecnología.

La función de la institución educativa como cohesionadora de grupos y promotora de valores se ve resentida, en alguna ocasión, por la difícil convivencia manifiesta, especialmente en los centros de educación secundaria; no obstante, se debe contextualizar los hechos desde una perspectiva global para enjuiciar adecuadamente las circunstancias que se muestran a la opinión pública, (Caballero, 2010). El Tema de la violencia en las instituciones educativas ha sido un tema 
de preocupación en la opinión pública desde hace varios años atrás y que se ha tratado de disminuirlo de diferentes maneras; sin embargo, aún siguen latentes en las instituciones educativas ocasionando diversos problemas a niños y jóvenes en los establecimientos educativos, (Palomo, 2017).

Lamentablemente las variables de convivencia escolar y solución de conflictos son una problemática que va en aumento, debido a los altos índices de casos reportados por las instituciones educativas, este ha sido un tema que ha generado diversas investigaciones. El estudio fue realizado en la Institución Educativa Departamental John F. Kennedy sede Cauca del municipio de Aracataca, es común observar manifestaciones violentas y comportamientos erráticos por parte de los estudiantes en la interacción con su grupo de pares, donde se evidencian agresiones verbales, físicas, discriminación, lenguaje soez, ruptura de normas escolares e irrespeto a la autoridad, la cual es representada por docentes y cuerpo administrativo de la institución.

Partiendo de los comportamientos anteriormente mencionados, surge la necesidad de proponer estrategias pedagógicas que permitan fortalecer la convivencia escolar, generando espacios que propicien la solución de conflictos, mediante el uso de las herramientas TIC, donde a partir de metodologías innovadoras, se logre una transformación social. Las normas de convivencia, están basadas en los Derechos Humanos, considerando los valores innatos a los individuos, de ahí que hoy en día se les dé relevancia a algunas conductas "positivas" a una serie de cualidades del ser humano y que son estimadas como valores universales como; respeto, libertad, bondad, justicia, igualdad, amor, honradez, solidaridad, verdad, responsabilidad, amistad, valentía, honor y paz, éstos son objeto de estudio de la Ética, la Moral y la Filosofía.

Partiendo de lo anterior, el presente estudio buscó presentar estrategias pedagógicas para fortalecer la convivencia escolar y solución de conflictos, mediante el uso de las TIC en la Institución Educativa Departamental John F. Kennedy sede Cauca del municipio de Aracataca, promoviendo una educación pacifica, incluyente y humanizante, mediante las TIC como método innovador.

Convivencia escolar y solución de conflictos: una manera de mediar con las TIC

La convivencia escolar es entendida como la acción de vivir en compañía con otras personas en el contexto escolar de forma pacífica y armónica. Hace referencia al conjunto de relaciones interpersonales que ocurre entre las personas que conforman la comunidad educativa, dichas relaciones deben enfocarse en el logro de objetivos educativos y su desarrollo integral, (Garreton, 2014).

El conflicto nace de la confluencia de intereses o de la intersección de dos posiciones frente a una necesidad, situación, objeto o intención. El conflicto, como una situación de confrontación entre dos protagonistas, puede cursar con agresividad, cuando fallan, en alguna medida, los instrumentos mediadores con los que hay que enfrentarse al mismo. Así, cuando está en juego una tensión de intereses y aparece un conflicto, todo depende de los procedimientos y estrategias que se empleen para salir de él. Si se usan procedimientos belicosos, aparecerán episodios agresivos, que pueden cursar con violencia, (Ortega, 2015). 
La conflictividad escolar se ha convertido en una de las mayores preocupaciones de la sociedad, como se evidencia en los artículos aparecidos en los medios de comunicación en los últimos años, a pesar de que las prácticas educativas son favorecedoras de una buena convivencia, y que las relaciones interpersonales que se dan en este ámbito, habitualmente son de cooperación, ayuda, estima, y aprecio, en las instituciones educativas se presentan casos concretos sobre agresiones o acoso/ denigración a compañeros, convirtiéndose en una de las principales preocupaciones de los docentes, por tanto, éstos son conscientes de la importancia de abordar el tema, (Caballero, 2010).

Según Colombo, (2011) La violencia en las escuelas tiene la forma de un auténtico iceberg, de ninguna manera se trata de accidentes fortuitos y aleatorios, en consecuencia, no pueden abordarse y tratarse tampoco de manera aislada. Lo cierto es que los problemas de violencia no pueden abordarse sólo por vía represiva, debido a que esto puede multiplicarlos y hacerlos aún más graves. Por lo cual es responsabilidad de los centros educativos dar una respuesta esencialmente educativa a esos sucesos.

Por su parte, la solución de conflictos como estrategia de intervención pacífica, busca propiciar a la sociedad una cultura de paz, donde sea el diálogo y el entendimiento uno de las formas más utilizadas frente a un conflicto, pues solo de esta manera se proporcionará a la sociedad herramientas de comunicación y paz, generando desde la escuela espacios de participación que promuevan una sociedad democrática, justa, capaz de proponer soluciones asertivas a las situaciones conflictivas, (Palomo, 2017).

Por su parte Vásquez, (2012) menciona que el término resolución de conflic- tos induce a creer que de lo que se trata es de eliminar los conflictos, percibido como un estado negativo con necesidad de erradicarlo, la solución se basa en el arribo a un acuerdo final que evite y anule la situación conflictiva. Por otro lado, según Herrera, (2016) Las TIC se han convertido en una herramienta innovadora en el contexto educativo generando una relación ineludible que se puede aplicar a cualquier modelo pedagógico a desarrollar y a tener presente de forma trasversal como instrumento pedagógico.

En efecto, los conflictos son impulsados por una o más de las tres motivaciones subyacentes: competitiva, cooperativa e independiente. La primera lleva a intentar obtener lo máximo posible a costa del otro. La segunda invoca la norma "todos ganamos", es decir, que el otro también consiga lo máximo que sea posible. La tercera implicaría tomar decisiones prescindiendo de la otra parte, en la búsqueda de los propios objetivos. Se podría decir que esta última se fomenta en el sistema capitalista actual razonado en el ideal del éxito material y acumulación de capital en beneficio propio. Más aún, en el sistema educativo se tiende a exaltar este fin para el cual es necesario esforzarse y llegar a "ser alguien" en la vida, como sinónimo de obtener una profesión que permita alcanzar a mayores beneficios que la generación de los propios padres.

Según quienes investigan, en raras ocasiones los conflictos son meramente competitivos, cooperativos o individualistas. Casi perennemente son situaciones en las que se combinan una serie de motivaciones, fundamentalmente en aquellos conflictos caracterizados por la interdependencia. Si dos personas tienen señal que la otra quiere o desea, se necesita que cooperen a pesar de que cada una trate de obtener lo máximo posible. 
Todo conflicto en cualquier nivel, puede llegar a una de las dos grandes categorías de solución: acuerdo y resolución. El acuerdo se refiere a un cambio comportamental que ocurre cuando ambas partes encuentran una vía para llegar a un consenso, pero su posición actitudinal básica permanece sin cambio. La resolución implica tanto un cambio en el comportamiento como una convergencia en las actitudes subyacentes. Un cambio comportamental sin cambio actitudinal ocurre cuando una parte se somete a la demanda de la otra, pero continúa sintiéndose coaccionada.

Por su parte, la resolución del conflicto implica un cambio actitudinal mutuo que lleva a las partes a internalizar un nuevo patrón de interacción. Es indicar, el acuerdo ocurre porque las actitudes de los disputantes se han modificado. Las instituciones y su ejercicio no solo son entidades externas, sino que son incorporadas en la imparcialidad de los individuos que las componen, lo cual aumenta su impacto e influencia sobre sus comportamientos. A la vez, (Palomo, 2017), hace referencia que toda institución educativa se ve transitada por los siguientes elementos:

a. Un conjunto amplio de seres humanos y su particular forma de organización, que les permite asegurar las necesidades básicas para subsistir.

b. Un conjunto de producciones culturales que procuran sostener esta organización porque ha demostrado ser útil para un cierto fin.

c. Un proceso que posibilita este sostén, la socialización, y otros que acuden a apoyarlo en sus efectos, los del control social.

d. Una tensión inevitable entre los deseos de los individuos y la necesidad de amoldarlos a la forma social admitida. e. Otro conjunto de producciones culturales destinadas a convencer y generar la decisión que exige el sacrificio del deseo individual en pro de la estabilidad colectiva.

Ahora, esto resulta realmente complejo si se piensa que el conjunto social no es homogéneo en cuanto a oportunidades y su entramado tiende a encubrir la violencia que implica la expropiación de derechos en el origen de esas diferencias y las establece como un orden natural. Otra tensión inevitable es la que se configura entre los grupos y sectores con diferente posición respecto de esos derechos especiales.

Cabe decir que, desde este nivel de la descripción, las instituciones que preservan el sostenimiento del conjunto social que son maneras de preservar la esencial forma como se ha distribuido el poder para el uso privilegiado de bienes económicos, sociales y culturales. Es particular la fuerza con que se ocultan y rechazan los conflictos de poder y la dimensión política que toda institución contiene. Incluso al concebir la escuela como "el segundo hogar", se la está caracterizando como un espacio libre de conflicto. Cuando las instituciones se singularizan en la forma de una unidad organizacional concreta, en ellas el sujeto encuentra, por un lado, seguridad, pertenencia y desarrollo; y por otro, la enajenación, la exclusión y el sufrimiento. El conflicto se hace evidente en la medida en que las instituciones educativas sufren la incidencia de mandatos sociales de carácter paradójico.

Sin embargo, se critica a los teóricos de la expansión al sugerir que pensar la escuela como un espacio donde solo se coarta la libertad y se reproduce un orden social determinado pasa a cumplir la función de ocultar su real potencial de 
cambio. Las escuelas son instituciones que encierran todas las paradojas de la vida social, de ahí surge la calidad de describir y desentrañar las contradicciones, dinamismos y conflictos que se manifiestan en ella para utilizar ese conocer en pro de la desalienación.

Es así como surge, en la época moderna, la preocupación por una protección integral a los estudiantes, observándolos como sujetos potenciales en nuevas acciones de política legislativa en casi todos los países del mundo, lo que ha generado la consagración de los derechos, a través de preceptos, valores y principios constitucionales, generando un análisis científico. (Herrera, 2013).

Por su parte, la inclusión de las TIC en la educación ha llevado a los docentes a replantearse sus roles, estos influyen directamente en el proceso enseñanzaaprendizaje, y dichos cambios o avances que se han presentado a través del tiempo en el proceso educativo han permitido que se utilicen las herramientas tecnológicas como material de apoyo para el desarrollo de estrategias innovadoras con la finalidad generar motivación en los estudiantes, factor importante y necesario para fomentar un aprendizaje significativo, (Acuña, 2003).

En la actualidad las nuevas tecnologías de la información y comunicación (TIC) han tomado un gran auge y su integración al contexto educativo ha generado grandes cambios en el proceso de enseñanza, estas giran en torno a tres medios básicos: la informática, la microelectrónica y las telecomunicaciones; de manera interactiva e interconectadas, lo que permite conseguir nuevas realidades comunicativas, impactando de manera positiva la escuela.

Las TIC son tecnologías para almacenar, recuperar, procesar y comunicar la información. Existe una variedad de recursos electrónicos que se encuadran dentro del concepto de TIC como; televisión, teléfonos, videos, ordenadores, entre otros. Sin embargo, los medios más representativos de la sociedad actual son los ordenadores que nos permiten utilizar diferentes aplicaciones informáticas presentaciones, aplicaciones multimedia, programas ofimáticos y redes de comunicación.

La sociedad escolar en Colombia enfrenta retos en lo económico, social, político y cultural, para construir un país abierto a las nuevas realidades de la ciencia y el conocimiento, exigiendo el desarrollo y fomento de capacidades para que sus ciudadanos puedan afrontar los cambios de estos tiempos. Ante tal premisa se hace necesario la apropiación social y conformación de comunidades, a fin de atender a las necesidades de la población y resuelva sus problemas, teniendo en cuenta las potencialidades naturales, culturales y particularidades de la región.

Así mismo, es urgente construir una convivencia plena, lo que sólo será posible si la mayoría de los educandos accede a los procesos de generación de conocimiento y saber, participando en ellos a través de mecanismos que les permitan interesarse, comprenderlos, validarlos y reconocerlos operando en su vida cotidiana; es decir, formarse una opinión al respecto y participar como ciudadanos plenos en la toma de decisiones sobre su vida, sobre los problemas que los afecten directa o indirectamente y sobre todos los aspectos de la existencia en los que incidan la ciencia y la tecnología.

No obstante, el uso de las TIC no implica la implementación de una estrategia de enseñanza/aprendizaje. En algunos casos se producen procesos edu- 
cativos que integran las TIC siguiendo una metodología tradicional en la que se enfatiza el proceso de enseñanza, donde el estudiante recibe la información del docente. No obstante, para guiar el proceso educativo, se puede fomentar la interacción, el aprendizaje colaborativo y el aprendizaje por descubrimiento utilizando las TIC basados en los diferentes recursos y servicios que ofrece Internet.

La apropiación social de la ciencia, tecnología e innovación, habla de la transferencia e intercambio del conocimiento, la participación ciudadana en CTI, la comunicación CTS, y la gestión del conocimiento para la apropiación, originando la apropiación social del conocimiento de la ciencia, tecnología e innovación; evitando que se muestre la ciencia como una actividad de un único actor, insistiendo en las relaciones que conforman las redes del saber más que en individualismo de la raza humana, propiciando espacios para la creatividad y de co-construcción.

Al hacer referencia a la apropiación social de la ciencia, la tecnología y la innovación, pretende apoyar el diseño y la implementación de estrategias de apropiación de la CTI, que muestren un diálogo efectivo entre expertos en ciencia, la tecnología y comunidades, en la generación y el uso del conocimiento para la solución de problemas específicos. En el marco de prácticas como responsabilidad social empresarial, transferencia tecnológica y del conocimiento, diálogo de saberes, innovación social, compromiso y comprensión pública y extensión universitaria, distintos actores, tradiciones e instituciones advierten sobre la importancia de comprender el conocimiento en términos de co-producción, co-creación y colaboración, impactando notoriamente la apropiación social y conformación de comunidades.
En este sentido, la convivencia escolar y solución de conflictos mediada por las Tic, permiten promover la cultura de las relaciones interpersonales o grupales que se generan en una escuela, adquiere relevancia de ser analizada; pues ésta contiene los elementos indispensables para lograr comprender el posicionamiento más ordenado en torno a la educación mencionado anteriormente. Esto significa que la convivencia sirve de escenario para analizar y comprender en qué medida las prácticas educativas se están dirigiendo o no hacia su ideal: la construcción y apoderamiento de un saber significativo para cada educando. Es decir que, podemos advertir que esta estructura de interacciones sociales está entrando en conflicto, dado el complejo contexto que vive la escuela actualmente.

\section{Metodología}

\section{Diseño}

La metodología utilizada en el estudio es de tipo cualitativo, entendida como una categoría de diseños de investigación que extraen descripciones a partir de observaciones que adoptan la forma de entrevistas, narraciones, notas de campo, grabaciones, transcripciones de audio, vídeo, registros escritos de todo tipo, fotografías o películas y artefactos. Pérez (2012). A su vez se utilizó el modelo de investigación acción que es una metodología de investigación orientada a la práctica educativa.

Las características principales de este modelo son la transformación y mejora de una realidad escolar, partiendo de la perspectiva de quien posee el problema, implica la colaboración de participantes, requiere la reflexión sis- 
temática en la acción, convirtiendo la práctica en objeto de investigación, formando parte del mismo proceso, esta es realizada por las personas implicadas en la investigación, donde el elemento de "formación" es esencial en el proceso investigativo.

\section{Procedimiento}

Para presentar estrategias pedagógicas para fortalecer la convivencia escolar y solución de conflictos, mediante el uso de tecnologías de la información y la comunicación (TIC) en básica primaria, la metodología respondió a una serie de etapas:

- Etapa (1): selección y análisis de la población sujeto de estudio.

- Etapa (2): integración de la temática, se integraron grupos estudiantiles para seleccionar problemáticas relacionadas el contexto escolar. Los estudiantes se plantearon preguntas y con el acompañamiento del docente realizaron el proceso de indagación mediante herramientas virtuales y entrevistas a la comunidad.

- Etapa (3): socialización de los resultados de las indagaciones de estudiantes en el aula de clase.

- Etapa (4): análisis de los resultados para observar la influencia de variables contextuales en los procesos académico de los estudiantes.

- Etapa (5): divulgación de los resultados.

\section{Resultados}

Después de revisar cada uno de los componentes que anteceden la convivencia escolar y la solución de conflictos, este último término, desde un punto de vista positivo, es el motor de cambio social y sus efectos, siempre que sepamos gestionarlo bien, permiten establecer relaciones cada vez más cooperativas. El estudio de los conflictos y la forma de gestionarlos de modo cooperativo son, sin lugar a dudas, el elemento principal de cualquier educación que pretenda construir o fabricar la paz. Por eso, la resolución no violenta de los conflictos ha construido uno de los campos a mejorar en los procesos de enseñanza actuales y de acción de la investigación mediante las tecnologías de la información sobre la paz de los movimientos alternativos.

Al presentar estrategias pedagógicas para fortalecer la convivencia escolar y solución de conflictos, mediante el uso de tecnologías de la información y la comunicación (TIC) en básica primaria, se presentaron algunos factores, que inciden significativamente para su desarrollo como:

f. La diversidad creciente de funciones que debe desempeñar la escuela, sobre todo en su rol socializador.

g. Su empobrecimiento en cuanto a recursos.

h. Su desactualización en la posesión y uso de herramientas del conocimiento e información actuales.

i. Su desconexión con otros cambios en la sociedad actual.

j. La tensión constante frente a la evaluación de los medios de comunicación, que se centran en sus errores.

k. Las distancias generacionales entre adultos y jóvenes.

1. Las diferencias socioculturales de sus actores. 
En efecto, la convivencia escolar es una problemática que va en aumento, debido a los altos índices de casos reportados por las instituciones educativas, este ha sido un tema que ha generado diversas investigaciones. El estudio fue realizado en la Institución Educativa Departamental John F. Kennedy sede Cauca del municipio de Aracataca, en esta es común observar manifestaciones violentas y comportamientos erráticos por parte de los estudiantes en la interacción con su grupo de pares, donde se evidencian agresiones verbales, físicas, discriminación, lenguaje soez, desavenencia de normas escolares e irrespeto a la autoridad, la cual es representada por docentes y cuerpo administrativo de la institución.

Además, los procesos de comprensión e intervención de las relaciones entre tecno-ciencia y sociedad, construido desde la participación activa de los diversos grupos sociales que generan conocimiento, tributa a la convivencia escolar y solución de conflictos, mediante el uso de tecnologías de la información y la comunicación (TIC), amplía las dinámicas de producción de conocimiento más allá de las sinergias entre sectores académicos, productivos, y estatales, considerando las siguientes características: (ver figura 1)

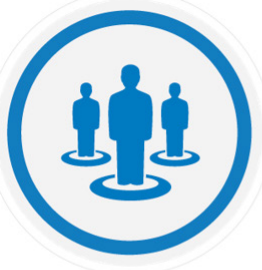

La investigación es organizada e intencionada.

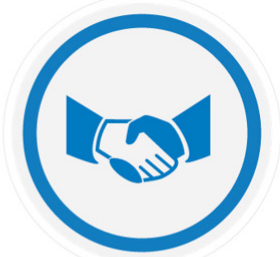

La red socio-técnica que lo constituye intervienen grupos sociales, expertos en ciencia y tecnología.

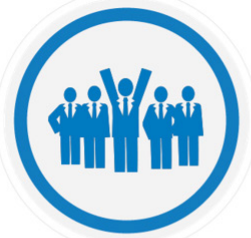

Proceso donde la sociedad civil se empodera a partir del conocimiento.

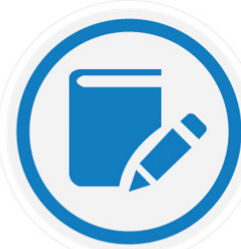

Apropiación no es enajenación.

Figura 1. Características de la convivencia escolar y solución de conflictos, mediante el uso de la TIC Fuente: elaboración propia (2018). 
Tabla 1

Estrategias para la convivencia escolar y solución de conflictos mediadas por las TIC

\begin{tabular}{|c|c|}
\hline Estrategias & Indicador \\
\hline Dominación. & $\begin{array}{l}\text { Ocurre cuando una de las partes intenta imponer sus deseos por medios físicos } \\
\text { o psicológicos, lo cual implica el uso de la violencia. Esto tiene directa relación } \\
\text { con la posición que ocupan los disputantes en la estructura de poder. }\end{array}$ \\
\hline Capitulación. & $\begin{array}{l}\text { Consiste en que una de las partes cede en forma unilateral ante la otra, } \\
\text { independiente de la magnitud de sus demandas. Puede resultar de la creencia } \\
\text { del que toma decisiones de que tiene pocas opciones. }\end{array}$ \\
\hline Retirada. & $\begin{array}{l}\text { Sucede cuando una parte abandona el conflicto, negándose a seguir formando } \\
\text { parte de él por más tiempo. }\end{array}$ \\
\hline Inactividad. & $\begin{array}{l}\text { La inactividad se da cuando una parte no hace nada, deliberadamente, esperando } \\
\text { que el tiempo de paso a una solución. }\end{array}$ \\
\hline Negociación. & $\begin{array}{l}\text { Se realiza cuando dos o más partes interdependientes usan el sistema de oferta } \\
\text { y contraoferta, buscando un acuerdo que resulte mutuamente aceptable. }\end{array}$ \\
\hline $\begin{array}{l}\text { Intervención de } \\
\text { terceras partes. }\end{array}$ & $\begin{array}{l}\text { Método en el cual un individuo o grupo que no es parte del conflicto, es decir, } \\
\text { que representa imparcialidad para las partes involucradas, interviene para } \\
\text { ayudar a identificar los problemas y avanzar hacia un acuerdo. En Psicología } \\
\text { Social, se llama a este proceso, la mediación, práctica que ha sido más } \\
\text { desarrollada especialmente en ámbitos judiciales, de terapia familiar y en } \\
\text { diversas organizaciones. Se tiende a utilizar en casos en que la intensidad del } \\
\text { conflicto es baja y sólo necesaria cuando las partes han intentado buscar una } \\
\text { solución sin tener éxito. }\end{array}$ \\
\hline
\end{tabular}

Fuente: elaboración propia (2018).

El conflicto sobre determinados temas puede estar obscurecido por problemas en otros niveles y esto ocurre tanto a nivel interpersonal, intergrupal, inter organizacional e internacional, por tal motivo se hace necesario promover estrategias convivencia escolar y solución de conflictos (ver tabla 1).

\section{Conclusiones}

Partiendo de los comportamientos anteriormente mencionados, surge la necesidad de proponer estrategias pedagógicas que permitan fortalecer la convivencia escolar, generando espacios que propicien la solución de conflictos, mediante el uso de las herramientas TIC, donde a partir de metodologías innovadoras, se logre una transformación social, en la Institución Educativa Departamental John F. Kennedy sede Cauca del mu- nicipio de Aracataca, promoviendo una educación pacifica, incluyente y humanizante, mediante las TIC como método innovador.

Al presentar estrategias pedagógicas para fortalecer la convivencia escolar y solución de conflictos, se concluye que las tecnologías de la información y comunicación, en los procesos de enseñanza-aprendizaje se han proliferado en los últimos años a través de internet e incorporación cada vez mayor de diferentes recursos y herramienta de acceso distinto al computador, posibilitando su incorporación en la educación y le han dado una nueva dimensión al aprendizaje. De hecho, tiene que ver con el deseo del ser humano de mantenerse instruido y actualizado a lo largo de su vida no solo a través de escenarios de formación presenciales sino también a través de escenarios de formación por medios virtuales, que 
les permita acceder a la información y al conocimiento desde cualquier lugar y cualquier hora con unas condiciones técnicas de acceso básicas.

Por tal motivo se debe generar un marco estratégico que destaca la necesidad de fortalecer el acceso a la educación preescolar, básica y media con calidad orientada desde una perspectiva de atención integral con ambientes educativos especializados y pertinentes, apoyados en sistemas de información y registro detallado de los niños, niñas y adolescentes, enriquecido con el mejoramiento continuo de los procesos pedagógicos, la cualificación de los agentes educativos, el aseguramiento de la calidad y la necesidad de responder al reto de una atención integral que contemple las necesidades y propuestas regionales, con el uso de las TIC.

Las tecnologías se han convertido en una herramienta para el desarrollo del hombre y la satisfacción de algunas necesidades, este proceso ha estado guiado por la visión que las nuevas Tecnologías de la Información y la Comunicación tienen el potencial de alterar el escenario donde se introducen y, por tanto, pueden facilitar la exploración y reformulación de conocimientos que antes eran de difícil acceso, lo que ha generado una práctica activa, impulsando cambios para cualificar las condiciones estructurales del sector educativo en cuanto cobertura y calidad. Es así como estas herramientas no sólo han facilitado la digitalización de la información, sino que también permiten conectar al estudiante a un ordenador o un servidor que a través del cual pueden acceder a toda clase de información en los diferentes modos de la red y desarrollar competencias investigativas para una educación de calidad.

\section{Referencias bibliográficas}

Acuña, A. (2003). La robótica educativa: un motor para la innovación. Fundación Omar Dengo San José, Costa Rica

Caballero, M. (2010). Convivencia escolar. Un estudio sobre buenas prácticas. Revista Paz y Conflictos. Número 3.

Casassus, J. (2003). La escuela y la (des) igualdad. Santiago: Ediciones LOM.

Colombo, G. (2011). Violencia Escolar y Convivencia Escolar: Descubriendo estrategias en la vida cotidiana escolar. Revista Argentina de Sociología, vol. 8-9, núm. 15-16, Consejo de Profesionales en Sociología. Buenos Aires, Argentina.

Funes, G. (2013). Conflicto en las organizaciones y mediación. Universidad Internacional de Andalucía. Guías pedagógicas para la convivencia escolar. (2013) Ley 1620 de 2013 - Decreto 1965 de 2013.

Garreton Valdivia, P. (2014). Estado de la convivencia escolar, conflictividad y su forma de abordarla en establecimientos educacionales de alta vulnerabilidad social de la provincia de Concepción, Chile.

Herrera Tapias, B. (2013). La constitucionalización de los derechos del consumidor en Colombia: un análisis desde los derechos sociales fundamentales. Civilizar. Ciencias Sociales y Humanas, 13 (25), 33-47.

Herrera-Tapias, B. (2016). Cultura Ciudadana y las Tecnologías de la Información y la Comunicación. Editorial YOYOBIZ. Barranquilla-Colombia.

Mena, M. y Huneeus, M. (2017). Convivencia Escolar para el aprendizaje y buen trato de todos: hacia una mejor comprensión del concepto. Cultura. Educación y Sociedad 8(2), 9-20. 
Ortega, R. (2015). La Convivencia Escolar: qué es y cómo abordarla. Consejería de Educación y Ciencia.

Palomo, E. (2017). Solución de Conflictos. Tesis de para optar Título de Licenciado en Ciencias de la Educación mención Educación Básica.
Pérez, G. (2012). Competencias o pensamiento práctico. La construcción de los significados de representación y de acción. Educar por competencias ¿qué hay de nuevo? (2aed., pp. 59-102). Madrid, España: Ediciones Morata.

Vásquez, A. (2012). Resolución de conflictos. Bogotá: El planeta. (p. 14) 